\title{
Akuter Schlaganfall Thrombolyse ist bei Nachweis eines Gefäßverschlusses wirksamer
}

De Silva DA, Churilov L, Olivot JM et al; on behalf of the EPITHET-DEFUSE Investigators. Greater effect of stroke thrombolysis in the presence of arterial obstruction. Ann Neurol 2011; 70: $601-5$
Fragestellung: Ist die systemische Thrombolyse mit Gewebeplasminogenaktivator bei Patienten mit nachgewiesenem Gefäßverschluss wirksamer als bei fehlendem Nachweis?

Hintergrund: Ziel der systemischen Thrombolyse mit rekombinanten Gewebeplasminogenaktivator (rt-PA) ist die Rekanalisation eines verschlossenen Gefäßes im Bereich der hirnversorgenden Arterien oder im intrakraniellen Kreislauf. Die meisten Studien die zur Zulassung der Thrombolyse geführt haben, hatten allerdings keine CT- oder Kernspinangiografie durchgeführt, sodass nicht bekannt ist, wie hoch die Rekanalisationsrate wirklich ist, und ob Patienten mit nachgewiesenem Gefäßverschluss mehr von einer systemischen Thrombolyse profitieren, als Patienten ohne entsprechenden Nachweis.

Patienten und Methodik: Die Auswertung basiert auf den Daten von zwei prospektiven Therapiestudien. EPITHET war eine multizentrische randomisierte Studie, in der Patienten nach multimodaler MR-Bildgebung intravenös mit rt-PA oder Placebo in einem Zeitfenster von drei bis sechs Stunden behandelt wurden. Die DEFUSE-Studie war eine multizentrische prospektive Kohortenstudie, bei der die Patienten in einem Zeitfenster von drei bis sechs Stunden ebenfalls mit Thrombolyse behandelt wurden. In beiden Studien wurden bei den Patienten beim Studieneinschluss eine Kernspintomografie inklusive Kernspinangiografie, sowie diffusionsgewichteter und perfusionsgewichteter Bildgebung durchgeführt. In der DEFUSE-Studie wurde die Größe der endgültigen Läsion in der Kernspintomografie nach 30 Tagen und in der EPITHET-Studie nach 90 Tagen bestimmt; hier wurden T2-gewichtete Sequenzen verwen- det. Die Kernspinangiografie wurde verblindet ausgewertet. Die Gefäßbefunde wurden in freie Gefäße, partiellen und kompletten Verschluss eingeteilt.

Ergebnisse: Von den 101 Patienten der EPITHET- und den 174 der DEFUSE-Studie lagen 154 auswertbare Kernspintomografien vor. Bei 116 Patienten standen auch Daten zur endgültigen Infarktgröße zur Verfügung. Von diesen 116 Patienten hatten 72 zum Zeitpunkt des Studieneinschlusses einen Gefäßverschluss und 44 offene Gefäße. Bei 45 Patienten lag ein kompletter und bei 27 ein partieller Gefäßverschluss vor. Die Verschlüsse waren in der Arteria carotis interna bei 22 Patienten, in der Arteria cerebri media im M1-Abschnitt bei 43 Patienten, in der Arteria cerebri media im M2-Abschnitt bei vier Patienten und in der Arteria cerebri posterior bei drei Patienten lokalisiert. Patienten mit einem nachgewiesenen Verschluss waren jünger, hatten deutlich schwerere Schlaganfälle, ein signifikant größeres Infarktvolumen und ein deutlich größeres Mismatch-Volumen als Patienten ohne Gefäßverschluss. Bei Patienten mit Gefäßverschluss nahm die Infarktgröße bei einer Thrombolyse signifikant weniger zu, als bei Placebo. Bei Patienten ohne Gefäßverschluss war die Zunahme des Infarktvolumens bei Placebo und rt-PA gleich.

Schlussfolgerungen: Bei Patienten mit akutem Schlaganfall, die für eine Thrombolyse innerhalb eines Zeitfensters von drei bis sechs Stunden ausgewählt werden, ist die Größenzunahme des Infarkts bei nachgewiesenem Gefäßverschluss unter einer Behandlung mit rt-PA signifikant geringer, als unter Placebo. Bei Patienten ohne Gefäßverschluss hat die systemische Thrombolyse offenbar keinen therapeutischen Nutzen.
Kommentar: Ziel der Thrombolyse ist es letztendlich, ein verschlossenes Gefäß zu eröffnen. Ein wichtiger Kritikpunkt an den bisher durchgeführten Zulassungsstudien zur Thrombolyse ist zweifelsfrei, dass in den meisten dieser Studien der Gefäßstatus nicht bekannt war. Dabei ist anzunehmen, dass viele Patienten einer Thrombolyse unterzogen wurden, bei denen entweder bereits eine spontane Rekanalisierung eingetreten war, oder bei denen ein so kleines Gefäß verschlossen war, dass es sich in der Computertomografie- oder Kernspinangiografie nicht darstellen ließ. Nimmt man die Ergebnisse dieser Post-hoc-Analyse aus Australien ernst, sollte tatsächlich jenseits des 3-Stunden-Zeitfensters überlegt werden, ob nicht doch routinemäßig eine CTAngiografie beim akuten ischämischen Insult durchgeführt wird, und bevorzugt die Patienten die einer systemischen oder lokalen Thrombolyse unterzogen werden, bei denen tatsächlich ein Gefäßverschluss nachweisbar ist. Da die Ergebnisse auf einer Post-hoc-Analyse beruhen, müsste dieses Konzept allerdings noch in einer prospektiven randomisierten Studie belegt werden.

Hans-Christoph Diener, Essen 